\title{
Linguistic Fingerprints of Internet Censorship: the Case of Sina Weibo
}

\author{
Kei Yin Ng, Anna Feldman, Jing Peng \\ Montclair State University \\ Montclair, New Jersey, USA
}

\begin{abstract}
This paper studies how the linguistic components of blogposts collected from Sina Weibo, a Chinese microblogging platform, might affect the blogposts' likelihood of being censored. Our results go along with King et al. (2013)'s Collective Action Potential (CAP) theory, which states that a blogpost's potential of causing riot or assembly in real life is the key determinant of it getting censored. Although there is not a definitive measure of this construct, the linguistic features that we identify as discriminatory go along with the CAP theory. We build a classifier that significantly outperforms non-expert humans in predicting whether a blogpost will be censored. The crowdsourcing results suggest that while humans tend to see censored blogposts as more controversial and more likely to trigger action in real life than the uncensored counterparts, they in general cannot make a better guess than our model when it comes to 'reading the mind' of the censors in deciding whether a blogpost should be censored. We do not claim that censorship is only determined by the linguistic features. There are many other factors contributing to censorship decisions. The focus of the present paper is on the linguistic form of blogposts. Our work suggests that it is possible to use linguistic properties of social media posts to automatically predict if they are going to be censored.
\end{abstract}

\section{Introduction}

In 2019, Freedom in the World ${ }^{1}$, a yearly survey produced by Freedom House ${ }^{2}$ that measures the degree of civil liberties and political rights in every nation, recorded the 13th consecutive year of decline in global freedom. This decline spans across long-standing democracies such as USA as well as authoritarian regimes such as China and Russia. "Democracy is in retreat. The offensive against freedom of expression is being supercharged by a new and more effective form of digital authoritarianism." According to the report, China is now exporting its model of comprehensive internet censorship and surveillance around the world, offering trainings, seminars, and even study trips as well as advanced equipment.

\footnotetext{
Copyright (c) 2020, Association for the Advancement of Artificial Intelligence (www.aaai.org). All rights reserved.

${ }^{1}$ https://freedomhouse.org/report/freedom-world/

freedom-world-2019/democracy-in-retreat

${ }^{2}$ https://freedomhouse.org/
}

In this paper, we deal with a particular type of censorship - when a post gets removed from a social media platform semi-automatically based on its content. We are interested in exploring whether there are systematic linguistic differences between posts that get removed by censors from Sina Weibo, a Chinese microblogging platform, and the posts that remain on the website. Sina Weibo was launched in 2009 and became the most popular social media platform in China. Sina Weibo has over 431 million monthly active users ${ }^{3}$.

In cooperation with the ruling regime, Weibo sets strict control over the content published under its service (Tager, Bass, and Lopez 2018). According to Zhu et al. (2013), Weibo uses a variety of strategies to target censorable posts, ranging from keyword list filtering to individual user monitoring. Among all posts that are eventually censored, nearly $30 \%$ of them are censored within 5-30 minutes, and nearly 90\% within 24 hours (Zhu et al. 2013). We hypothesize that the former are done automatically, while the latter are removed by human censors.

Research shows that some of the censorship decisions are not necessarily driven by the criticism of the state (King, Pan, and Roberts 2013), the presence of controversial topics (Ng, Feldman, and Leberknight 2018; Ng et al. 2018), or posts that describe negative events ( $\mathrm{Ng}$ et al. 2019). Rather, censorship is triggered by other factors, such as for example, the collective action potential (King, Pan, and Roberts 2013), i.e., censors target posts that stimulate collective action, such as riots and protests.

The goal of this paper is to compare censored and uncensored posts that contain the same sensitive keywords and topics. Using the linguistic features extracted, a neural network model is built to explore whether censorship decision can be deduced from the linguistic characteristics of the posts.

The contributions of this paper are: 1 . We decipher a way to determine whether a blogpost on Weibo has been deleted by the author or censored by Weibo. 2. We develop a corpus of censored and uncensored Weibo blogposts that contain sensitive keyword(s). 3. We build a neural network classifier that predicts censorship significantly better than non-expert humans. 4. We find a set of linguistics features

\footnotetext{
${ }^{3}$ https://www.investors.com/news/technology/ weibo-reports-first-quarter-earnings/
} 
that contributes to the censorship prediction problem. 5. We indirectly test the construct of Collective Action Potential (CAP) proposed by King et al. (2013) through crowdsourcing experiments and find that the existence of CAP is more prevalent in censored blogposts than uncensored blogposts as judged by human annotators.

\section{Previous Work}

There have been significant efforts to develop strategies to detect and evade censorship. Most work, however, focuses on exploiting technological limitations with existing routing protocols (Leberknight, Chiang, and Wong 2012; Katti, Katabi, and Puchala 2005; Levin et al. 2015; McPherson, Shokri, and Shmatikov 2016; Weinberg et al. 2012). Research that pays more attention to linguistic properties of online censorship in the context of censorship evasion include, for example, Safaka et al. (2016) who apply linguistic steganography to circumvent censorship. Lee (2016) uses parodic satire to bypass censorship in China and claims that this stylistic device delays and often evades censorship. Hiruncharoenvate et al. (2015) show that the use of homophones of censored keywords on Sina Weibo could help extend the time a Weibo post could remain available online. All these methods rely on a significant amount of human effort to interpret and annotate texts to evaluate the likelihood of censorship, which might not be practical to carry out for common Internet users in real life. There has also been research that uses linguistic and content clues to detect censorship. Knockel et al. (2015) and Zhu et al. (2013) propose detection mechanisms to categorize censored content and automatically learn keywords that get censored. King et al. (2013) in turn study the relationship between political criticism and chance of censorship. They come to the conclusion that posts that have a Collective Action Potential get deleted by the censors even if they support the state. Bamman et al. (2012) uncover a set of politically sensitive keywords and find that the presence of some of them in a Weibo blogpost contribute to a higher chance of the post being censored. $\mathrm{Ng}$ et al. (2018) also target a set of topics that have been suggested to be sensitive, but unlike Bamman et al. (2012), they cover areas not limited to politics. $\mathrm{Ng}$ et al. (2018) investigate how the textual content as a whole might be relevant to censorship decisions when both the censored and uncensored blogposts include the same sensitive keyword(s).

Our work is related to $\mathrm{Ng}$ et al. (2018) and $\mathrm{Ng}$ et al. (2019); however, we introduce a larger and more diverse dataset of censored posts; we experiment with a wider range of features and in fact show that not all the features reported in $\mathrm{Ng}$ et al. guarantee the best performance. We built a classifier that significantly outperforms $\mathrm{Ng}$ et al. We conduct a crowdsourcing experiment testing human judgments of controversy and censorship as well as indirectly testing the construct of collective action potential proposed by King et al.

\section{Tracking Censorship}

Tracking censorship topics on Weibo is a challenging task due to the transient nature of censored posts and the scarcity of censored data from well-known sources such as FreeWeibo ${ }^{4}$ and WeiboScope ${ }^{5}$. The most straightforward way to collect data from a social media platform is to make use of its API. However, Weibo imposes various restrictions on the use of its API such as restricted access to certain endpoints and restricted number of posts returned per request. Above all, the Weibo API does not provide any endpoint that allows easy and efficient collection of the target data (posts that contain sensitive keywords). Therefore, an alternative method is needed to track censorship for our purpose.

\section{Datasets \\ Using Zhu et al. (2003)'s Corpus}

Zhu et al. (2013) collected over 2 million posts published by a set of around 3,500 sensitive users during a 2-month period in 2012. We extract around 20 thousand text-only posts using 64 keywords across 26 topics, which partially overlap with those included in the New Corpus (see below and in Table 3). We filter all duplicates. Among the extracted posts, $930(4.63 \%)$ are censored by Weibo as verified by Zhu et al. (2013) The extracted data from Zhu et al.(2013)'s are also used in building classification models.

While it is possible to study the linguistic features in Zhu et al's dataset without collecting new data, we created another corpus that targets 'normal' users (Zhu et al. target 'sensitive' users) and a different time period so that the results are not specific to a particular group of users and time.

\section{New Corpus}

Web Scraping We develop a web scraper that continuously collects and tracks data that contain sensitive keywords on the front-end. The scraper's target interface ${ }^{6}$ displays 20 to 24 posts that contain a certain search key term(s), resembling a search engine's result page. We call this interface the Topic Timeline since the posts all contain the same keyword(s) and are displayed in reverse chronological order. The Weibo API does not provide any endpoint that returns the same set of data appeared on the Topic Timeline. Through a series of trial-and-errors to avoid CAPTCHAs that interrupt the data collection process, we found an optimal scraping frequency of querying the Topic Timeline every 5 to 10 minutes using 17 search terms (see Appendix ${ }^{7}$ ) across 10 topics (see Table 1) for a period of 4 months (August 29, 2018 to December 29, 2018). In each query, all relevant posts and their meta-data are saved to our database. We save posts that contain texts only (i.e. posts that do not contain images, hyperlinks, re-blogged content etc.) and filter out duplicates.

Decoding Censorship According to Zhu et al. (2013), the unique ID of a Weibo post is the key to distinguish whether

\footnotetext{
${ }^{4}$ https://freeweibo.com/

${ }^{5}$ http://weiboscope.jmsc.hku.hk/

${ }^{6}$ e.g. searching "NLP" http://s.weibo.com/weibo/NLP

${ }^{7}$ https://msuweb.montclair.edu/ feldmana/publications/ aaai20_appendix.pdf
} 


\begin{tabular}{c|c|c} 
topic & censored & uncensored \\
\hline cultural revolution & 55 & 66 \\
\hline human rights & 53 & 71 \\
\hline family planning & 14 & 28 \\
\hline censorship \& propaganda & 32 & 56 \\
\hline democracy & 119 & 107 \\
\hline patriotism & 70 & 105 \\
\hline China & 186 & 194 \\
\hline Trump & 320 & 244 \\
\hline Meng Wanzhou & 55 & 76 \\
\hline kindergarten abuse & 48 & 5 \\
\hline Total & $\mathbf{9 5 2}$ & $\mathbf{9 5 2}$
\end{tabular}

Table 1: Data collected by scraper for classification

a post has been censored by Weibo or has been instead removed by the authors themselves. If a post has been censored by Weibo, querying its unique ID through the API returns an error message of "permission denied" (systemdeleted), whereas a user-removed post returns an error message of "the post does not exist" (user-deleted). However, since the Topic Timeline (the data source of our web scraper) can be accessed only on the front-end (i.e. there is no API endpoint associated with it), we rely on both the front-end and the API to identify system- and user-deleted posts. It is not possible to distinguish the two types of deletion by directly querying the unique ID of all scraped posts because, through empirical experimentation, uncensored posts and censored (system-deleted) posts both return the same error message - "permission denied"). Therefore, we need to first check if a post still exists on the front-end, and then send an API request using the unique ID of the post that no longer exists to determine whether it has been deleted by the system or the user. The steps to identify censorship status of each post are illustrated in Figure 1. First, we check whether a scraped post is still available through visiting the user interface of each post. This is carried out automatically in a headless browser 2 days after a post is published. If a post has been removed (either by system or by user), the headless browser is redirected to an interface that says "the page doesn't exist"; otherwise, the browser brings us to the original interface that displays the post content. Next, after 14 days, we use the same methods in step 1 to check the posts' status again. This step allows our dataset to include posts that have been removed at a later stage. Finally, we send a follow-up API query using the unique ID of posts that no longer exist on the browser in step 1 and step 2 to determine censorship status using the same decoding techniques proposed by Zhu et al. as described above (2013). Altogether, around 41 thousand posts are collected, in which 952 posts $(2.28 \%)$ are censored by Weibo. In our ongoing work, we are comparing the accuracy of the classifier on posts that are automatically removed vs. those removed by humans. The results will be reported in the future publications.

We would like to emphasize that while the data collection methods could be seen as recreating a keyword search, the scraping pipeline also deciphers the logic in discovering censorship on Weibo.

\section{Sample Data}

Figure 2 shows several examples selected randomly from our dataset. Each pair of censored and uncensored posts contains the same sensitive keyword.

\section{Crowdsourcing Experiment}

A balanced corpus is created. The uncensored posts of each dataset are randomly sampled to match with the number of their censored counterparts (see Table 1 and Table 3). We select randomly a subset of the data collected by the web scraper to construct surveys for crowdsourcing experiment. The surveys ask participants three questions (see Figure 3).

Sample questions are included in Appendix ${ }^{8}$. Question 1 explores how humans perform on the task of censorship classification; question 2 explores whether a blogpost is controversial; question 3 serves as a way to explore in our data the concept of Collective Action Potential (CAP) suggested by King et al.(2013). According to King et al. (2013), Collective Action Potential is the potential to cause collective action such as protest or organized crowd formation outside the Internet. Participants can respond either Yes or No to the 3 questions above. A total of 800 blogposts (400 censored and 400 uncensored) are presented to 37 different participants through a crowdsourcing platform Witmart ${ }^{9}$ in 8 batches (100 blogposts per batch). Each blogpost is annotated by 6 to 12 participants. The purpose of this paper is to shed light on the "knowledge gap" between censors and normal weibo users about censorable content. We believe weibo users are aware of potential censorable content but are not "trained" enough to avoid or identify them. The results are summarized in Table 2.

\begin{tabular}{l|c|c} 
question & censored & uncensored \\
\hline \multirow{2}{*}{ Q1 (censorship) } & Yes: $23.83 \%$ & Yes: $16.41 \%$ \\
& No: $76.17 \%$ & No: $83.59 \%$ \\
\hline \multirow{2}{*}{ Q2 (controversy) } & Yes: $61.02 \%$ & Yes: $55.37 \%$ \\
& No: $38.98 \%$ & No: $44.63 \%$ \\
\hline \multirow{2}{*}{ Q3 (CAP) } & Yes: $6.13 \%$ & Yes: $3.93 \%$ \\
No: $93.87 \%$ & No: $96.07 \%$ \\
\hline
\end{tabular}

Table 2: Crowdsourcing results

The annotation results are intuitive - participants tend to see censored blogposts as more controversial and more likely to trigger action in real life than the uncensored counterpart.

We obtain a Fleiss's kappa score for each question to study the inter-rater agreement. Since the number and identity of participants of each batch of the survey are different, we obtain an average Fleiss' kappa from the results of each batch. The Fleiss' kappa of questions 1 to 3 are $0.13,0.07$, and 0.03 respectively, which all fall under the category of slight agreement.

We hypothesize that since all blogposts contain sensitive keyword(s), the annotators choose to label a fair amount of

\footnotetext{
${ }^{8}$ http://msuweb.montclair.edu/ feldmana/publications/ aaai20_appendix.pdf

${ }^{9}$ http://www.witmart.com
} 


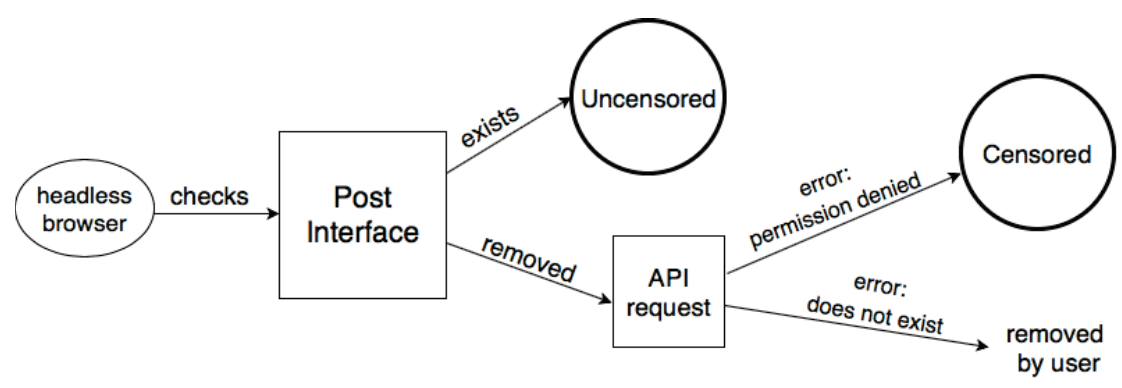

Figure 1: Logical flow to determine censorship status (Ng et al. 2018)

censored: 地铁站内走着, 两个台湾女孩大声聊着 台湾选举, 感受到一丝丝民主的气息 (Walking in the subway station, I heard two Taiwanese girls discussing freely about the election in Taiwan. I could feel a spirit of democracy.)

uncensored: “美国始终相信，台湾对民主的拥抱为所 有华人展示了一条更好的道路。”我对这句话真是念念 不忘。("The US still believes that Taiwan's welcoming of democracy shows all the Chinese a path to a better society" - I just cannot forget this remark.)

censored: 这两年微博舆论环境真的是文革吧。 我支持 $\mathrm{A}$ 知错改过依法认罪 $=$ 我支持 $\mathrm{A}$ 犯法, 是这 个逻辑吗? (The public opinions on Weibo in the past 2 years were like the denouncement made during Cultural Revolution - I support A to admit and fix his mistakes and plead guilty legitimately $=\mathrm{I}$ support A to commit crimes. Is this the logic?)

uncensored: 最近的一系列事情大概只能让人感慨一句 文革从未走远吧 (The series of events happened recently only made one felt that Cultural Revolution has never really left us.)

Figure 2: Sample Data

uncensored blogposts as controversial, and even as likely to be censored or cause action in real life. This might also be the reason of the low agreement scores - the sensitive keywords might be the cause of divided opinions.

Regarding the result of censorship prediction, 23.83\% of censored blogposts are correctly annotated as censored, while $83.59 \%$ of uncensored blogposts are correctly annotated as uncensored. This result suggests that participants tend to predict a blogpost to survive censorship on Weibo, despite the fact that they can see the presence of controversial element(s) in a blogpost as suggested by the annotation results of question 2 . This suggests that detecting censorable content is a non-trivial task and humans do not have a good intuition (unless specifically trained, perhaps) what material is going to be censored. It might be true that there is some level of subjectivity form human censors. We believe there are commonalities among censored blogposts that pass through the "subjectivity filters" and such commonalities could be the linguistic features that contribute to our experiment results (see sections and).
Q1. Do you think this blogpost will be censored on Weibo? ( 你认为此内容会被微博屏蔽吗? )

Q2. Do you think this blogpost will trigger a debate? (你认 为此微博内容会触发争论或骂战吗? )

Q3. Do you think this blogpost might possibly trigger an assembly, a protest, or a riot? (你认为此微博内容有触发 集会、游行示威或暴动的可能吗?)

Figure 3: Crowdsourcing experiment: Three survey questions.

\section{Feature Extraction}

To build an automatic classifier, we first extract features from both our scraped data and Zhu et al.'s dataset. While the datasets we use are different from that of $\mathrm{Ng}$ et al. (2018) and $\mathrm{Ng}$ et al. (2019), some of the features we extract are similar to theirs. We include CRIE features (see below) and the number of followers feature that are not extracted in $\mathrm{Ng}$ et al. (2018)'s work.

\section{Linguistic Features}

We extract 5 sets of linguistic features from both datasets (see below) - the LIWC features, the CRIE features, the sentiment features, the semantic features, and word embeddings. We are interested in the LIWC and CRIE features because they are purely linguistic, which aligns with the objective of our study. Also, some of the LIWC features extracted from Ng et al. (2018)'s data have shown to be useful in classifying censored and uncensored tweets.

LIWC features The English Linguistic Inquiry and Word Count (LIWC) (Pennebaker, Booth, and Francis 2017; Pennebaker et al. 2015) is a program that analyzes text on a word-by-word basis, calculating percentage of words that match each language dimension, e.g., pronouns, function words, social processes, cognitive processes, drives, informal language use etc. Its lexicon consists of approximately 6400 words divided into categories belong to different linguistic dimensions and psychological processes. LIWC builds on previous research establishing strong links between linguistic patterns and personality/psychological state. We use a version of LIWC developed for Chinese by Huang et al. (2012) to extract the frequency of word categories. Altogether we extract 95 features from LIWC. One 


\begin{tabular}{|c|c|c|}
\hline topic & censored & uncensored \\
\hline cultural revolution & 19 & 29 \\
\hline human rights & 16 & 10 \\
\hline family planning & 4 & 4 \\
\hline censorship \& propaganda & 47 & 38 \\
\hline democracy & 94 & 53 \\
\hline patriotism & 46 & 30 \\
\hline China & 300 & 458 \\
\hline Bo Xilai & 8 & 8 \\
\hline brainwashing & 57 & 3 \\
\hline emigration & 10 & 11 \\
\hline June 4th & 2 & 5 \\
\hline food \& env. safety & 14 & 17 \\
\hline wealth inequality & 2 & 4 \\
\hline protest \& revolution & 4 & 5 \\
\hline stability maintenance & 66 & 28 \\
\hline political reform & 12 & 9 \\
\hline territorial dispute & 73 & 75 \\
\hline Dalai Lama & 2 & 2 \\
\hline HK/TW/XJ issues & 2 & 4 \\
\hline political dissidents & 2 & 2 \\
\hline Obama & 8 & 19 \\
\hline USA & 62 & 59 \\
\hline communist party & 37 & 10 \\
\hline freedom & 12 & 11 \\
\hline economic issues & 31 & 37 \\
\hline Total & 930 & 930 \\
\hline
\end{tabular}

Table 3: Data extracted from Zhu et al. (2013)'s dataset for classification

important feature of the LIWC lexicon is that categories form a tree structure hierarchy. Some features subsume others.

Sentiment features We use BaiduAI ${ }^{10}$ to obtain a set of sentiment scores for each post. BaiduAI's sentiment analyzer is built using deep learning techniques based on data found on Baidu, one of the most popular search engines and encyclopedias in mainland China. It outputs a positive sentiment score and a negative sentiment score which sum to 1.

CRIE features We use the Chinese Readability Index Explorer (CRIE) (Sung et al. 2016), a text analysis tool developed for measuring the readability of a Chinese text based on the its linguistic components. Its internal dictionaries and lexical information are developed based on dominant corpora such as the Sinica Tree Bank. CRIE outputs 50 linguistic features (see Appendix ${ }^{11}$ ), such as word, syntax, semantics, and cohesion in each text or produce an aggregated result for a batch of texts. CRIE can train and categorize texts based on their readability levels. We use the textual-features analysis for our data and derive readability scores for each post in our data. These scores are mainly based on descriptive statistics.

\footnotetext{
${ }^{10} \mathrm{https}: / /$ ai.baidu.com

${ }^{11} \mathrm{http}: / /$ msuweb.montclair.edu/ feldmana/publications/ aaai20_appendix.pdf
}

Semantic features We use the Chinese Thesaurus developed by Mei (1984) and extended by HIT-SCIR ${ }^{12}$ to extract semantic features. The structure of this semantic dictionary is similar to WordNet, where words are divided into 12 semantic classes and each word can belong to one or more classes. It can be roughly compared to the concept of word senses. We derive a semantic ambiguity feature by dividing the number of words in each post by the number of semantic classes in it.

Frequency \& readability We compute the average frequency of characters and words in each post using Da $(2004)^{13}$ 's work and Aihanyu's CNCorpus ${ }^{14}$ respectively. For words with a frequency lower than 50 in the reference corpus, we count it as $0.0001 \%$. It is intuitive to think that a text with less semantic variety and more common words and characters is relatively easier to read and understand. We derive a Readability feature by taking the mean of character frequency, word frequency and word count to semantic classes described above. It is assumed that the lower the mean of the 3 components, the less readable a text is. In fact, these 3 components are part of Sung et al. (2015)'s readability metric for native speakers on the word level and semantic level.

Word embeddings Word vectors are trained using the word2vec tool (Mikolov et al. 2013a; 2013b) on 300,000 of the latest Chinese $\operatorname{articles}^{15}$ on Wikipedia. A 200dimensional vector is computed for each word of each blogpost. The vector average of each blogpost is the sum of word vectors divided by the number of vectors. The 200dimensional vector average are used as features for classification.

\section{Non-linguistic Features}

Followers The number of followers of the author of each post is recorded and used as a feature for classification.

\section{Classification}

Features extracted from the balanced datasets (See Table 1 and Table 3) are used for classifications. Although the amount of uncensored blogposts significantly outnumber censored in real-life, such unbalanced corpus might be more suitable for anomaly detection. All numeric values of the features have been standardized before classification. We use a multilayer perceptron (MLP) classifier to classify instances into censored and uncensored. A number of classification experiments using different combinations of features are carried out.

Best performances are achieved using the combination of CRIE, sentiment, semantic, frequency, readability and follower features (i.e. all features but LIWC and

\footnotetext{
${ }^{12}$ Harbin Institute of Technology Research Center for Social Computing and Information Retrieval.

${ }^{13} \mathrm{http}$ ///lingua.mtsu.edu/chinese-computing/statistics/

${ }^{14} \mathrm{http} / / /$ www.aihanyu.org/cncorpus/index.aspx

${ }^{15}$ https://dumps.wikimedia.org/zhwiki/latest/
} 


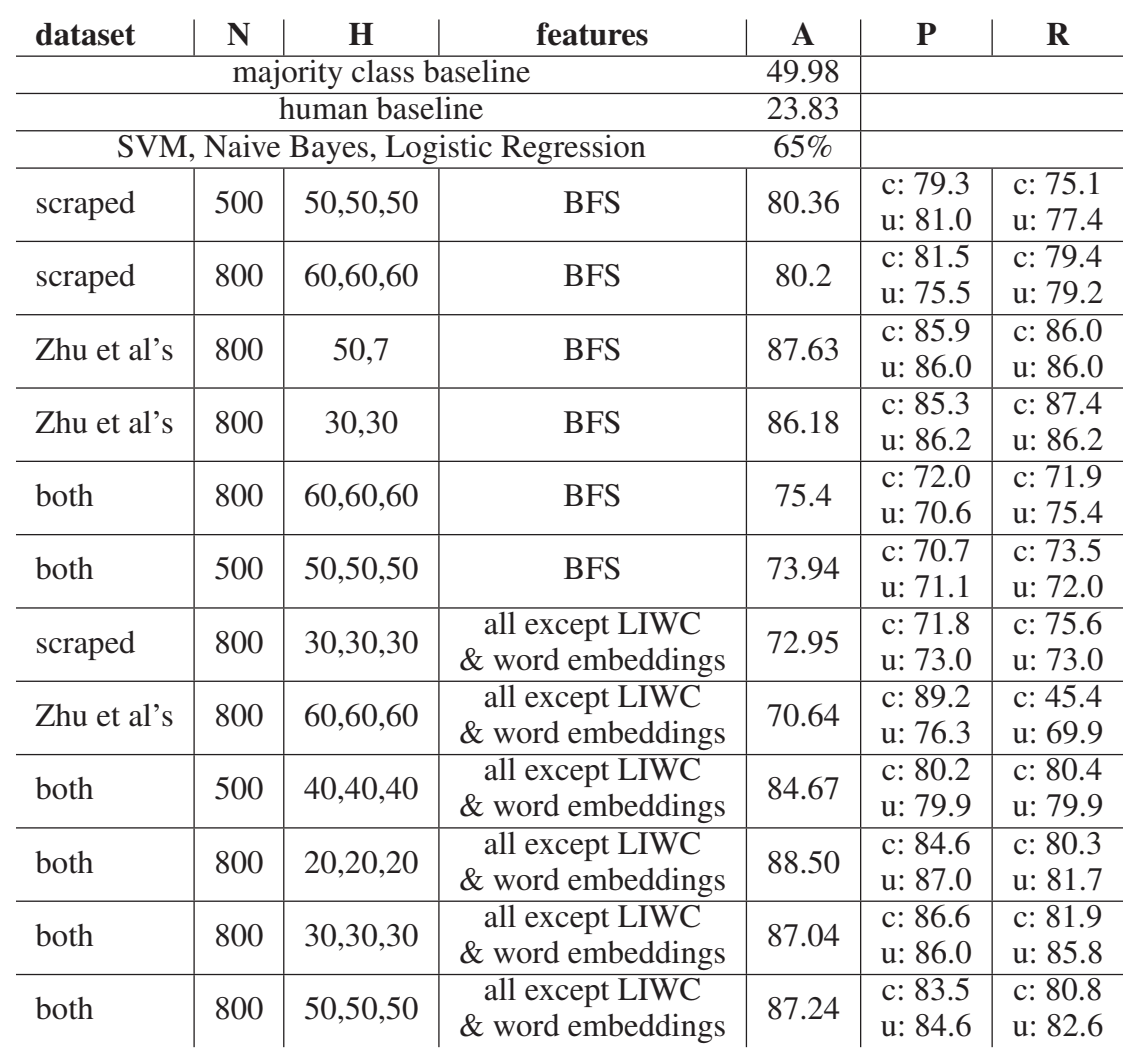

Table 4: MLP classification results. $\mathrm{N}=$ number of epochs, $\mathrm{H}=$ number of nodes in each hidden layer, $\mathrm{A}=$ accuracy, $\mathrm{P}=$ precision, $\mathrm{R}=$ recall, $\mathrm{BFS}=$ best features set, $\mathrm{c}=$ censored, $\mathrm{u}=$ uncensored

word embeddings) (see Table 4). The feature selection is performed using random sampling. As a result 77 features are selected that perform consistently well across the datasets. We call these features the best features set. (see https://msuweb.montclair.edu/ feldmana/publications/ aaai20_appendix.pdf for the full list of features).

We vary the number of epochs and hidden layers. The rest of the parameters are set to default - learning rate of 0.3 , momentum of 0.2 , batch size of 100 , validation threshold of 20 . Classification experiments are performed on 1) both datasets 2) scraped data only 3) Zhu et al.'s data only. Each experiment is validated with 10 -fold cross validation. We report the accuracy of each model in Table 4. It is worth mentioning that using the LIWC features only, or the CRIE features only, or the word embeddings only, or all features excluding the CRIE features, or all features except the LIWC and CRIE features all result in poor performance of below $60 \%$. Besides MLP, we also use the same sets of features to train classifiers using Naive Bayes, Logistic, and Support Vector Machine. However, the performances are all below $65 \%$.

\section{Discussion and Conclusion}

Our best results are over $30 \%$ higher than the baseline and about $60 \%$ higher than the human baseline obtained through crowdsourcing, which shows that our classifier has a greater censorship predictive ability compared to human judgments. The classification on both datasets together tends to give higher accuracy using at least 3 hidden layers. However, the performance does not improve when adding additional layers (other parameters being the same). Since the two datasets were collected differently and contain different topics, combining them together results in a richer dataset that requires more hidden layers to train a better model. It is worth noting that classifying both datasets using the best features set decreases the accuracy, while using all features but LIWC improves the classification performance. The reason for this behavior could be an existence of consistent differences in the LIWC features between the datasets. Since the LIWC features in the best features set (see Appendix https://msuweb.montclair.edu/ feldmana/ publications/aaai20_appendix.pdf) consist of mostly word categories of different genres of vocabulary (i.e. grammar and style agnostic), it might suggest that the two datasets use vocabularies differently. Yet, the high performance obtained excluding the LIWC features shows that the key to distinguishing between censored and uncensored posts seems to be the features related to writing style, readability, sentiment, and semantic complexity of a text.

Figure 5 shows two blogposts annotated by CRIE with number of verbs and number of first person pronoun features.

To narrow down on what might be the best features that contribute to distinguishing censored and uncensored posts, we compare the mean of each feature of the two classes 


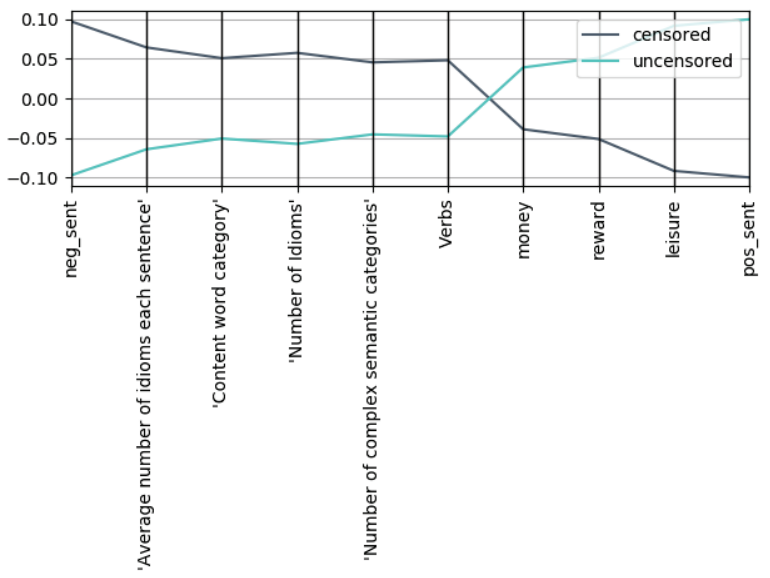

Figure 4: Parallel Coordinate Plots of the top 10 features that have the greatest difference in average values

censored: Verbs: 6 1st person pronouns: 2 这两年微博舆论环境真的是文革吧。我 支持 $A$ 知错 改 过依法 认罪 = 我 支持 $\mathrm{A}$ 犯法, 是这个逻辑吗? (The public opinions on Weibo in the past 2 years were like the denouncement made during Cultural Revolution - I support $A$ to admit and fix his mistakes and plead guilty legitimately $=\underline{I}$ support $\mathrm{A}$ to commit crimes. Is this the logic?)

uncensored: Verbs: 3 1st person pronouns: 0 最近的 一系列事情大概只能让人感溉一句文革从未走远吧 (The series of events happened recently only made one felt that Cultural Revolution has never really left us.)

Figure 5: Examples of blogposts annotated by CRIE.

(see Figure 4). The 6 features distinguish censored from uncensored are:

1. negative sentiment

2. average number of idioms in each sentence

3 . number of content word categories

4. number of idioms

5. number of complex semantic categories

6. verbs

On the other hand, the 4 features that distinguish uncensored from censored are:

1. positive sentiment

2. words related to leisure

3. words related to reward

4. words related to money

This might suggest that the censored posts generally convey more negative sentiment and are more idiomatic and semantically complex in terms of word usage. According to King et al. (2013), Collective Action Potential, which is re- lated to a blogpost's potential of causing riot or assembly in real-life, is the key determinant of a blogpost getting censored. Although there is not a definitive measure of this construct, it is intuitive to relate a higher average use of verbs to a post that calls for action.

On the other hand, the uncensored posts might be in general more positive in nature (positive sentiment) and include more content that talks about neutral matters (money, leisure, reward).

We further explore how the use of verbs might possibly affect censorship by studying the types of verbs used in censored and uncensored blogposts. We extracted verbs from all blogposts by using the Jieba Part-of-speech tagger ${ }^{16}$. We then used the Chinese Thesaurus described in Section to categorize the verbs into 5 classes: Actions, Psychology, Human activities, States and phenomena, and Relations. However, no significant differences have been found across censored and uncensored blogposts. A further analysis on verbs in terms of their relationship with actions and arousal can be a part of future work.

Since the focus of this paper is to study the linguistic content of blogposts, rather than rate of censorship, we did not employ technical methods to differentiate blogposts that have different survival rates. Future work could be done to investigate any differences between blogposts that get censored at different rates. In our ongoing work, we are comparing the accuracy of the classifier on posts that are automatically removed vs. those removed by humans. The results will be reported in the future publications.

To conclude, our work shows that there are linguistic fingerprints of censorship, and it is possible to use linguistic properties of a social media post to automatically predict if it is going to be censored. It will be interesting to explore if the same linguistic features can be used to predict censorship on other social media platforms and in other languages.

\footnotetext{
${ }^{16}$ https://github.com/fxsjy/jieba
} 


\section{Acknowledgments}

This work is supported by the National Science Foundation under Grant No.: 1704113, Division of Computer and Networked Systems, Secure and Trustworthy Cyberspace (SaTC). We also thank Jed Crandall for sharing Zhu et al. (2013)'s dataset with us.

\section{References}

Bamman, D.; O'Connor, B.; and Smith, N. A. 2012. Censorship and deletion practices in Chinese social media. First Monday 17(3).

Da, J. 2004. A corpus-based study of character and bigram frequencies in chinese e-texts and its implications for chinese language instruction. In Zhang, P.; Tianwei, X.; and $\mathrm{Xu}$, J., eds., The studies on the theory and methodology of the digitalized Chinese teaching to foreigners: Proceedings of the Fourth International Conference on New Technologies in Teaching and Learning Chinese., 501-511. Beijing: Tsinghua University Press.

Hiruncharoenvate, C.; Lin, Z.; and Gilbert, E. 2015. Algorithmically Bypassing Censorship on Sina Weibo with Nondeterministic Homophone Substitutions. In Ninth International AAAI Conference on Web and Social Media.

Huang, C.-L.; Chung, C.; Hui, N. K.; Lin, Y.-C.; Seih, Y.T.; Lam, B. C.; Chen, W.-C.; Bond, M.; and Pennebaker, J. H. 2012. The development of the chinese linguistic inquiry and word count dictionary. Chinese Journal of Psychology 54(2):185-201.

jiā jū Méi. 1984. The Chinese Thesaurus.

Katti, S.; Katabi, D.; and Puchala, K. 2005. Slicing the onion: Anonymous routing without pki. Technical report, MIT CSAIL Technical Report 1000.

King, G.; Pan, J.; and Roberts, M. E. 2013. How Censorship in China Allows Government Criticism but Silences Collective Expression. American Political Science Review 107(2):1-18.

Knockel, J.; Crete-Nishihata, M.; Ng, J.; Senft, A.; and Crandall, J. 2015. Every rose has its thorn: Censorship and surveillance on social video platforms in china. In Proceedings of the 5th USENIX Workshop on Free and Open Communications on the Internet.

Leberknight, C. S.; Chiang, M.; and Wong, F. M. F. 2012. A taxonomy of censors and anti-censors: Part i-impacts of internet censorship. International Journal of E-Politics (IJEP) $3(2)$.

Lee, S. 2016. Surviving online censorship in china: Three satirical tactics and their impact. China Quarterly.

Levin, D.; Lee, Y.; L.Valenta; amd V. Lai, Z. L.; Lumezanu, C.; Spring, N.; and Bhattacharjee, B. 2015. Alibi routing. In Proceedings of the 2015 ACM Conference on Special Interest Group on Data Communication.

McPherson, R.; Shokri, R.; and Shmatikov, V. 2016. Defeating image obfuscation with deep learning. arXiv preprint arXiv:1609.00408.
Mikolov, T.; Chen, K.; Corrado, G.; and Dean, J. 2013a. Efficient estimation of word representations in vector space. In Proceedings of Workshop at ICLR.

Mikolov, T.; Sutskever, I.; Chen, K.; Corrado, G.; and Dean, J. 2013b. Distributed representations of words and phrases and their compositionality. In Proceedings of NIPS.

Ng, K. Y.; Feldman, A.; Peng, J.; and Leberknight, C. 2018. Linguistic Characteristics of Censorable Language on SinaWeibo. In Proceedings of the 1st Workshop on NLP for Internet Freedom held in conjunction with COLING 2018.

Ng, K. Y.; Feldman, A.; Peng, J.; and Leberknight, C. 2019. Neural network prediction of censorable language. In Proceedings of the Third Workshop on Natural Language Processing and Computational Social Science, 40-46. Minneapolis, Minnesota: Association for Computational Linguistics.

Ng, K. Y.; Feldman, A.; and Leberknight, C. 2018. Detecting censorable content on sina weibo: A pilot study. In Proceedings of the 10th Hellenic Conference on Artificial Intelligence. ACM.

Pennebaker, J. W.; Boyd, R. L.; Jordan, K.; and Blackburn, K. 2015. The development and psychometric the development of psychometric properties of liwc. Technical report, University of Texas at Austin.

Pennebaker, J. W.; Booth, R.; and Francis, M. 2017. Linguistic Inquiry and Word Count (LIWC2007).

Safaka, I.; Fragouli, C.; ; and Argyraki, K. 2016. Matryoshka: Hiding secret communication in plain sight. In 6th USENIX Workshop on Free and Open Communications on the Internet (FOCI 16). USENIX Association.

Sung, Y.; Chang, T.; Lin, W.; Hsieh, K.; and Chang, K. 2015. Crie: An automated analyzer for chinese texts. Behavior Research Method.

Sung, Y.-T.; Chang, T.-H.; Lin, W.-C.; Hsieh, K.-S.; and Chang, K.-E. 2016. Crie: An automated analyzer for chinese texts. Behavior Research Methods 48(4):1238-1251.

Tager, J.; Bass, K. G.; and Lopez, S. 2018. Forbidden feeds: Government controls on social media in china. Technical report, Pen America.

Weinberg, Z.; Wang, J.; Yegneswaran, V.; Briesemeister, L.; Cheung, S.; Wang, F.; and Boneh, D. 2012. Stegotorus: A camouflage proxy for the tor anonymity system. Proceedings of the 19th ACM conference on Computer and Coтmunications Security.

Zhu, T.; Phipps, D.; Pridgen, A.; Crandall, J. R.; and Wallach, D. S. 2013. The velocity of censorship: High-fidelity detection of microblog post deletions. In Proceedings of the 22Nd USENIX Conference on Security, SEC'13, 227-240. Berkeley, CA, USA: USENIX Association. 